\title{
ANTIBACTERIAL ACTIVITY OF DOMESTIC BALKAN DONKEY MILK TOWARD Listeria monocytogenes AND Staphylococcus aureus
}

\author{
Ljubiša Ć. Šarić1 ${ }^{1 *}$, Bojana M. Šarić ${ }^{1}$, Snežana Ž. Kravić ${ }^{2}$, Dragana V. Plavšić1 ${ }^{1}$ Ivan Lj. Milovanović ${ }^{1}$, \\ Jasmina M. Gubić ${ }^{1}$, Nataša M. Nedeljković \\ ${ }^{1}$ University of Novi Sad, Institute of Food Technology, Bulevar cara Lazara 1, \\ 21000 Novi Sad, Serbia \\ ${ }^{2}$ University of Novi Sad, Faculty of Technology, Bulevar cara Lazara 1, 21000 Novi Sad, Serbia
}

\author{
*Corresponding author: \\ Phone: +381214853822 \\ Fax: +38121 450-725 \\ e-mail address: ljubisa.saric@fins.uns.ac.rs,
}

\begin{abstract}
The aim of this study was to investigate the antibacterial activity of raw milk from Domestic Balkan donkey breed toward Listeria monocytogenes and Staphylococcus aureus. Examination of antibacterial activity was performed in artificially contaminated milk samples by monitoring the changes of count of viable cells of tested bacteria during 8 hours of incubation at $38^{\circ} \mathrm{C}$. Lysozyme and fatty acids contents were also determined in donkey milk. The obtained results indicated inhibitory effect of donkey milk toward both tested bacteria. The lysozyme content in the analyzed milk samples was ranged from 0.67 to $3.54 \mathrm{~g} / \mathrm{L}$. The most abundant fatty acids with known antibacterial activity toward Gram positive bacteria were linoleic, lauric and oleic acid.
\end{abstract}

Key words: donkey milk, antibacterial activity, L. monocytogenes, S. aureus

\section{INTRODUCTION}

Donkey milk (DM) has been an object of scientific research during the last few years, although it has been known as a natural remedy for a long time. In recent times, its beneficial effects in the prevention of atherosclerosis (Tafaro et al., 2007), as well as its antibacterial (Šarić et al., 2012; Tidona et al. 2011; Zhang et al., 2008) antiviral (Brumini et al., 2013), antiproliferative and anti-tumor properties (Mao et al., 2009) received scientific confirmation. However, majority of investigations have been directed to its application in diet of infants and children allergic to cow's milk proteins (lacono et al., 1992; Monti et al., 2007; Vita et al., 2007). The DM possesses protein and lipid profiles similar to those of human milk, with low casein content and high percentage of essential amino acids (Fantuz et al., 2001; Salimei et al., 2004; Vincenzetti et al., 2008).

Donkey breeding is widespread in Africa, Asia and Eastern Europe (Blench, 1999; Kugler et al., 2008; Tadesse, 2010; Zhang et al., 2008). The Domestic Balkan donkey is primarily farmed in the Northern and Eastern regions of country (FAO DAD-IS, 2009; Kugler et al., 2008). The Special Nature Reserve "Zasavica", located in the northwest of Serbia, currently has over 100 female donkeys belonging to the Domestic Balkan donkey breed.

In Serbia, DM has been traditionally used in the treatment of asthma and bronchitis. Increasing demand for DM on the Serbian market is a consequence of the fact that 
number of estimated deaths by respiratory diseases in Serbia is higher than Europe and world average (ECSCEE, 2013). Local consumers in regions where donkeys are traditionally bred usually consume raw milk in order to avoid thermal degradation of the valuable substances. However, this practice inevitably raises the question of the safety of its consuming. Although some literature sources reported strong antibacterial activity of DM, the majority of these reports are related to its activity toward Gram negative bacteria members of the Enterobacteriaceae (Šarić et al., 2012; Tidona et al., 2011; Zhang et al., 2008). Gram positive food borne pathogens such as Listeria monocytogenes and Staphylococcus aureus can grow in wide variety of foods including raw milk. Patogenic bacteria present in raw milk may originate from milking animals or from environment during the collection or storage of the milk (FASFC, 2013). Limited literature data reported the absence of $L$. monocytogenes in raw DM (Sarno et al., 2012), while $S$. aureus was detected in low count only in a small number of samples (Conte et al., 2004). Although lysozyme (LYZ) is designated as the main anti-microbial agent in DM owing to its high concentration (Coppola et al., 2002; Vincenzetti et al., 2008; Zhang et al., 2008), some fatty acids which have already been determined in DM (Salimei \& Fantuz, 2012) could be important for overal antibacterial activity of DM toward Gram positive bacteria.

The object of this study was to investigate antimicrobial potential of raw Domestic Balkan donkey milk against $L$. monocytogenes and $S$. aureus. The content of LYZ as well as fatty acid composition with an emphasis on the fatty acids with known antimicrobial activity were also examined.

\section{MATERIALS AND METHODS}

\section{Sample collection}

Milk samples were obtained from Domestic Balkan donkey breed, from "Zasavica" Special Nature Reserve, Serbia. Eight clinically healthy donkeys from different lactation periods $(65-210$ days postpartum (pp.) were included in the trial. Samples collected after morning hand- milking, were immediately cooled at $4{ }^{\circ} \mathrm{C}$ and transported to the laboratory where the samples were frozen at $-20{ }^{\circ} \mathrm{C}$. The samples marked as $A, B, C$ were collected in the early stage (65-90 days pp.), samples $F, G$ in the middle stage (140-160 days $\mathrm{pp}$.) and samples $\mathrm{D}, \mathrm{E}, \mathrm{H}$ in the late stage of lactation (190-210 days pp.).

\section{Determination of protein profile}

Sample preparation was carried out according to Tidona et al. (2011) with some modifications. Milk samples were diluted in 1:1.5 (v/v), sample:buffer $(0.125 \quad \mathrm{M}$ TriseHCl, 4\% SDS, 2\% glycerol, $2 \%$ bmercaptoethanol, $\mathrm{pH}$ 6.8) and heated at $100{ }^{\circ} \mathrm{C}$ for $5 \mathrm{~min}$. The chip-based separations were performed on the Agilent 2100 bioanalyzer (Agilent Technologies, Santa Clara, CA, USA) in combination with the Protein 80 Plus LabChip kit and the dedicated Protein 80 software assay on 2100 expert software. Chips were prepared according to the protocol provided by the Protein 80 LabChip kit. Bovine serum albumin was used as the standard for quantitation of the milk proteins. All samples were analyzed in triplicate.

\section{Determination of fatty acid composition}

A modified method of Havemose et al. (2004) was used for the extraction of fat from DM. Fat was extracted by using methanol and chloroform. The mixture was shaken for $1 \mathrm{~min}$ and then centrifuged for $10 \mathrm{~min}$ at $3000 \mathrm{rpm}$. The lower phase containing the lipid fraction was isolated and evaporated to dryness under stream of nitrogen. The methylation of fatty acid extracted from DM was performed according to Kravić et al. (2010) with minor modifications. Previously extracted fats were dissolved in $2.4 \mathrm{~cm}^{3}$ of hexane. An aliquot $\left(0.6 \mathrm{~cm}^{3}\right)$ of $2 \mathrm{~mol} \mathrm{dm}$ methanolic $\mathrm{KOH}$ solution was added. The tube was capped and shaked for $20 \mathrm{~s}$ and allowed to boil one min in water bath at $70{ }^{\circ} \mathrm{C}$. After $20 \mathrm{~s}$ of shaking $1.2 \mathrm{~cm}^{3}$ of $1 \mathrm{~mol} \mathrm{dm}^{-3}$ $\mathrm{HCl}$ was added and gently stirred. After phase separation the upper phase containing the fatty acid methyl esters was decanted and $2 \mu \mathrm{l}$ was used for further analysis. The obtained samples were analyzed by a Hewlett-Packard (HP) 5890 gas chromatograph coupled with a HP 5971A 
mass spectrometer detector. A fused silica capillary column (Supelco) was used for the FAME analysis; SP-2560, $100 \mathrm{~m} \times$ $0.25 \mathrm{~mm}$ i.d., $0.20 \mu \mathrm{m}$ film thickness. The carrier gas ultra-pure helium was passed through moisture and oxygen traps with constant flow rate of $0.58 \mathrm{~cm}^{3} / \mathrm{min}$. The GC oven temperature was programmed from $100{ }^{\circ} \mathrm{C}(5 \mathrm{~min})$, to $240{ }^{\circ} \mathrm{C}$ at the rate of $6{ }^{\circ} \mathrm{C} / \mathrm{min}$, held at $240{ }^{\circ} \mathrm{C}$ for $10 \mathrm{~min}$. The injection temperature was $230{ }^{\circ} \mathrm{C}$ and the injection volume was $2.0 \mu \mathrm{l}$, split ratio 1:30. The mass spectrometer was operated in the electron ionization mode with quadruple temperature of $180{ }^{\circ} \mathrm{C}$. Data acquisition was carried out in the scan mode (range $40-400 \mathrm{~m} / \mathrm{z}$ ). The fatty acids peaks were identified by comparison of their retention times with retention times of standards from Supelco 37 component fatty acid methyl ester mix (Bellefonte, PA, USA). Commercial data base of mass spectra Wiley was also used. The content of each fatty acid expressed by mass percentage is calculated according to equation:

$$
\frac{R_{i} \cdot A_{i}}{\sum R_{i} \cdot A_{i}} \cdot 100
$$

where $R_{i}$ is the response factor for each fatty acid normalized to palmitic acid methyl ester and $A_{i}$ the peak area of fatty acid methyl ester in the sample solution.

\section{Antibacterial assay}

The antibacterial assay was performed on each individual milk sample using Listeria monocytogenes ATCC 19111 and Staphylococcus aureus ATCC 25923. Cultures were stored on nutrient agar slants in a refrigerator at $4{ }^{\circ} \mathrm{C}$ and subcultured on fresh slants weekly. After overnight incubation on nutrient agar at $37{ }^{\circ} \mathrm{C}$, well-isolated colonies of each test microorganisms were selected and transferred with an inoculating loop to a tube of sterile saline and vortexed thoroughly. The density of the bacterial suspension was adjusted to the 0.5 McFarland standard using DEN-1 densitometer (Biosan, Riga, Latvia). Further decimal dilutions in sterile saline were prepared from initial suspensions. DM samples were artificially contaminated with these bacterial strains at the level of contamination of $10^{2} \mathrm{cfu} / \mathrm{mL}$. $25 \mathrm{~mL}$ of each artificially contaminated sample were placed into a sterile vessel and kept in water bath (Raypa, Spain) at $38 \pm 0.5{ }^{\circ} \mathrm{C}$ during $8 \mathrm{~h}$. Changes in the number of tested bacteria were monitored every hour according to the international standards (EN ISO, 2004; ISO, 2003). The results were expressed as $\log _{10} \mathrm{cfu} / \mathrm{mL}$. Non inoculated DM was used as negative control, while artificially contaminated nutrient broth $(\mathrm{Hi}-$ media, India) was used as positive control. All samples were analyzed in triplicate.

\section{Statistical analysis}

Results were expressed as mean along with standard deviations, of triplicate analyses for all measurements. Analysis of variance was followed by Duncan's multiple comparison tests using STATISTICA version 10 (StatSoft Inc., Tulsa, OK, USA).

\section{RESULTS AND DISCUSSION}

The observed significantly lower number of $L$. monocytogenes (Table 1 ) and $S$. aureus (Table 2 ) in tested DM compared to positive controls, after $8 \mathrm{~h}$ of incubation at $38{ }^{\circ} \mathrm{C}$, indicates the antibacterial activity of DM against these bacteria. After the first hour of incubation, $L$. monocytogenes count in all tested samples decreased and reached the lowest value. This number slightly increased during the next five hours. The count of this pathogen was not significantly different in comparison to its initial value at the end of the incubation. After the $2 \mathrm{~h}$ period of duration of lag phase of $L$. monocytogenes in nutrient broth, its number started to increase and after $8 \mathrm{~h}$ reached the value of $5.00 \mathrm{log}$ $\mathrm{cfu} / \mathrm{mL}$.

The count of $S$. aureus in most of the tested samples slowly increased or remained at almost constant level during the first $5 \mathrm{~h}$. After $8 \mathrm{~h}$ of incubation, count of $S$. aureus was in range from 3.00 to 3.92 , whereas this number in positive control was much higher (6.84 log cfu/mL).

The tested bacterial strains did not detect in negative controls. It was obvious that the antibacterial activity of DM toward $S$. aureus could be characterized as growth inhibitory in terms of the extension of its lag phase and growth slowing. 
Table 1.

Changes in count of L. monocytogenes ATCC $19111\left(\log _{10} \mathrm{cfu} / \mathrm{mL}\right)$ in raw donkey milk during $8 \mathrm{~h}$ at $38^{\circ} \mathrm{C}$

\begin{tabular}{|c|c|c|c|c|c|c|c|c|c|}
\hline \multirow{2}{*}{ 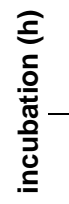 } & \multicolumn{9}{|c|}{ Donkey milk samples } \\
\hline & A & B & C & D & $\mathbf{E}$ & $\mathbf{F}$ & G & $\mathbf{H}$ & NB \\
\hline \multirow{2}{*}{0} & $2.30(0.05)$ & $2.31(0.04)$ & $2.32(0.03)$ & $2.30(0.04)$ & $2.33(0.02)$ & $2.30(0.02)$ & $2.34(0.05)$ & $2.30(0.03)$ & $2.33(0.04)$ \\
\hline & de,A & $\mathrm{C}, \mathrm{A}$ & $\mathrm{d}, \mathrm{A}$ & $\mathrm{d}, \mathrm{A}$ & $\mathrm{C}, \mathrm{A}$ & $\mathrm{f}, \mathrm{A}$ & $\mathrm{d}, \mathrm{A}$ & $\mathrm{e}, \mathrm{A}$ & $a, A$ \\
\hline \multirow{2}{*}{1} & $1.94(0.03)$ & $1.78(0.00)$ & $1.85(0.04)$ & $1.90(0.05)$ & $1.99(0.04)$ & $1.87(0.03)$ & $1.85(0.04)$ & $1.92(0.02)$ & $2.39(0.02)$ \\
\hline & a,DE & $a, A$ & a, B & $\mathrm{a}, \mathrm{BCD}$ & $a, E$ & $\mathrm{a}, \mathrm{BC}$ & a,B & a,CD & $b, F$ \\
\hline \multirow{2}{*}{2} & $2.08(0.04)$ & $2.04(0.18)$ & $2.07(0.06)$ & $2.10(0.08)$ & $2.00(0.05)$ & $2.11(0.04)$ & $2.02(0.07)$ & $2.00(0.05)$ & $2.38(0.02)$ \\
\hline & b,A & b,A & b,A & $\mathrm{C}, \mathrm{A}$ & $a b, A$ & $\mathrm{~b}, \mathrm{~A}$ & b,A & b,A & $a b, B$ \\
\hline \multirow{2}{*}{3} & $1.93(0.08)$ & $2.03(0.02)$ & $2.06(0.04)$ & $2.09(0.03)$ & $2.07(0.08)$ & $2.13(0.04)$ & $2.01(0.05)$ & $2.08(0.04)$ & $2.85(0.03)$ \\
\hline & a,A & b,B & $\mathrm{b}, \mathrm{BC}$ & $\mathrm{c}, \mathrm{BC}$ & $\mathrm{ab}, \mathrm{BC}$ & cde,C & $\mathrm{b}, \mathrm{AB}$ & $\mathrm{C}, \mathrm{BC}$ & C,D \\
\hline \multirow{2}{*}{4} & $2.25(0.07)$ & $1.97(0.12)$ & $2.06(0.09)$ & $2.07(0.05)$ & $2.10(0.09)$ & $2.07(0.06)$ & $2.10(0.08)$ & $2.11(0.06)$ & $3.37(0.01)$ \\
\hline & d, B & $b, A$ & $b, A$ & bc, $A$ & $b, A$ & bcd, $A$ & bc, $A$ & $\mathrm{C}, \mathrm{A}$ & d,C \\
\hline \multirow{2}{*}{5} & $2.10(0.05)$ & $1.95(0.00)$ & $2.02(0.04)$ & $2.03(0.05)$ & $2.05(0.06)$ & $2.15(0.08)$ & $1.99(0.10)$ & $2.09(0.00)$ & $3.80(0.00)$ \\
\hline & $\mathrm{bc}, \mathrm{CD}$ & $b, A$ & $b, A B C$ & $\mathrm{bc}, \mathrm{ABC}$ & $a b, A B C D$ & de,D & $b, A B$ & $c, B C D$ & e,E \\
\hline 6 & $\begin{array}{c}2.25(0.07) \\
d, D\end{array}$ & $\begin{array}{c}1.94(0.03) \\
b, A\end{array}$ & $\begin{array}{c}2.04(0.05) \\
\text { b,BC }\end{array}$ & $\begin{array}{c}1.98(0.06) \\
a, A B\end{array}$ & $\begin{array}{c}2.04(0.04) \\
a b, B C\end{array}$ & $\begin{array}{c}2.05(0.05) \\
\text { bc, BC }\end{array}$ & $\begin{array}{c}2.06(0.04) \\
\text { bc,BC }\end{array}$ & $\begin{array}{c}2.12(0.03) \\
C, C\end{array}$ & $\begin{array}{c}4.11(0.07) \\
f, E\end{array}$ \\
\hline \multirow{2}{*}{7} & $2.20(0.05)$ & $2.25(0.01)$ & $2.18(0.03)$ & $2.22(0.04)$ & $2.25(0.06)$ & $2.21(0.03)$ & $2.15(0.07)$ & $2.19(0.05)$ & $4.54(0.02)$ \\
\hline & $\mathrm{cd}, \mathrm{AB}$ & $\mathrm{C}, \mathrm{A}$ & $\mathrm{c}, \mathrm{AB}$ & $\mathrm{d}, \mathrm{AB}$ & $\mathrm{C}, \mathrm{A}$ & $\mathrm{e}, \mathrm{AB}$ & $\mathrm{C}, \mathrm{B}$ & $\mathrm{d}, \mathrm{AB}$ & g,C \\
\hline \multirow{2}{*}{8} & $2.37(0.10)$ & $2.27(0.08)$ & $2.30(0.03)$ & $2.28(0.06)$ & $2.33(0.04)$ & $2.32(0.07)$ & $2.35(0.03)$ & $2.26(0.04)$ & $5.00(0.01)$ \\
\hline & e,B & $\mathrm{c}, \mathrm{AB}$ & $\mathrm{d}, \mathrm{AB}$ & $\mathrm{d}, \mathrm{AB}$ & $\mathrm{C}, \mathrm{AB}$ & $\mathrm{f}, \mathrm{AB}$ & $\mathrm{d}, \mathrm{AB}$ & de,A & h,C \\
\hline
\end{tabular}

Table 2.

Changes in count of $S$. aureus ATCC $25923\left(\log _{10} \mathrm{cfu} / \mathrm{mL}\right)$ in raw donkey milk during $8 \mathrm{~h}$ at $38{ }^{\circ} \mathrm{C}$

\begin{tabular}{|c|c|c|c|c|c|c|c|c|c|}
\hline \multirow{2}{*}{ 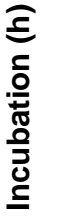 } & \multicolumn{9}{|c|}{ Donkey milk samples } \\
\hline & A & B & C & D & $\mathbf{E}$ & $\mathbf{F}$ & $\mathbf{G}$ & $\mathbf{H}$ & NB \\
\hline 0 & $2.32(0.05)$ & $2.30(0.05)$ & $2.35(0.05)$ & $2.34(0.05)$ & $2.33(0.05)$ & $2.32(0.04)$ & $2.34(0.05)$ & $2.33(0.05)$ & $2.30(0.05)$ \\
\hline 1 & $\begin{array}{c}2.43(0.04) \\
b, C D\end{array}$ & $\begin{array}{c}\text { a,A } \\
2.43(0.01) \\
b, C D\end{array}$ & $\begin{array}{c}\text { a,A } \\
2.34(0.09) \\
a, B C\end{array}$ & $\begin{array}{c}\mathrm{b}, \mathrm{A} \\
2.22(0.06) \\
\mathrm{b}, \mathrm{A}\end{array}$ & $\begin{array}{c}\text { ab,A } \\
2.46(0.10) \\
a b, D\end{array}$ & $\begin{array}{c}\mathrm{ab}, \mathrm{A} \\
2.27(0.04) \\
\mathrm{a}, \mathrm{AB}\end{array}$ & $\begin{array}{c}\text { a,A } \\
2.33(0.07) \\
a, A B C\end{array}$ & $\begin{array}{c}\mathrm{ab}, \mathrm{A} \\
2.26(0.01) \\
\mathrm{a}, \mathrm{AB}\end{array}$ & $\begin{array}{c}a, A \\
2.25(0.04) \\
a, A B\end{array}$ \\
\hline 2 & $\begin{array}{c}2.45(0.04) \\
b, C\end{array}$ & $\begin{array}{c}2.44(0.00) \\
b, C\end{array}$ & $\begin{array}{c}2.32(0.01) \\
a, B\end{array}$ & $\begin{array}{c}2.18(0.03) \\
a, A\end{array}$ & $\begin{array}{c}2.27(0.05) \\
a, A B\end{array}$ & $\begin{array}{c}2.25(0.07) \\
a, A B\end{array}$ & $\begin{array}{c}2.28(0.02) \\
a, B\end{array}$ & $\begin{array}{c}2.27(0.09) \\
a b, A B\end{array}$ & $\begin{array}{c}2.26(0.06) \\
a, A B\end{array}$ \\
\hline 3 & $\begin{array}{c}2.48(0.02) \\
b c, E\end{array}$ & $\begin{array}{c}2.44(0.02) \\
b, D E\end{array}$ & $\begin{array}{c}2.57(0.03) \\
b, F\end{array}$ & $\begin{array}{c}2.28(0.06) \\
a b, A\end{array}$ & $\begin{array}{c}2.35(0.03) \\
a b, B C\end{array}$ & $\begin{array}{c}2.41(0.03) \\
\quad c, C D\end{array}$ & $\begin{array}{c}2.28(0.06) \\
\mathrm{a}, \mathrm{A}\end{array}$ & $\begin{array}{c}2.35(0.04) \\
a b, B C\end{array}$ & $\begin{array}{c}2.30(0.00) \\
a, A B\end{array}$ \\
\hline 4 & $\begin{array}{c}2.52(0.04) \\
\text { bc, CE }\end{array}$ & $\begin{array}{c}2.56(0.02) \\
C, E\end{array}$ & $\begin{array}{c}2.60(0.12) \\
b, E F\end{array}$ & $\begin{array}{c}2.49(0.06) \\
\text { c,BCE }\end{array}$ & $\begin{array}{c}2.40(0.13) \\
a b, B C\end{array}$ & $\begin{array}{c}2.38(0.05) \\
\text { bc, AB }\end{array}$ & $\begin{array}{c}2.71(0.01) \\
b, F\end{array}$ & $\begin{array}{c}2.27(0.06) \\
a b, A\end{array}$ & $\begin{array}{c}2.70(0.00) \\
b, F\end{array}$ \\
\hline 5 & $\begin{array}{c}2.55(0.03) \\
\quad \mathrm{C}, \mathrm{BC}\end{array}$ & $\begin{array}{c}2.52(0.10) \\
c, B C\end{array}$ & $\begin{array}{c}3.21(0.06) \\
a, A B\end{array}$ & $\begin{array}{c}2.53(0.19) \\
c, B C\end{array}$ & $\begin{array}{c}2.75(0.05) \\
b, C\end{array}$ & $\begin{array}{c}2.70(0.01) \\
d, C\end{array}$ & $\begin{array}{c}2.71(0.21) \\
b, C\end{array}$ & $\begin{array}{c}2.26(0.24) \\
a, A\end{array}$ & $\begin{array}{c}3.71(0.02) \\
C, D\end{array}$ \\
\hline 6 & $\begin{array}{c}2.57(0.02) \\
\mathrm{C}, \mathrm{AB}\end{array}$ & $\begin{array}{c}2.68(0.05) \\
\text { d,AB }\end{array}$ & $\begin{array}{c}3.64(0.03) \\
c, C\end{array}$ & $\begin{array}{c}2.85(0.00) \\
d, B\end{array}$ & $\begin{array}{c}3.07(0.10) \\
b, B\end{array}$ & $\begin{array}{c}3.00(0.04) \\
e, B\end{array}$ & $\begin{array}{c}2.87(0.09) \\
b, B\end{array}$ & $\begin{array}{c}2.28(0.20) \\
a b, A\end{array}$ & $\begin{array}{c}4.39(0.09) \\
d, D\end{array}$ \\
\hline 7 & $\begin{array}{c}3.06(0.02) \\
d, B\end{array}$ & $\begin{array}{c}3.45(0.02) \\
e, D\end{array}$ & $\begin{array}{c}3.92(0.03) \\
d, F\end{array}$ & $\begin{array}{c}3.14(0.01) \\
e, B\end{array}$ & $\begin{array}{c}3.37(0.02) \\
\quad, C D\end{array}$ & $\begin{array}{c}3.33(0.03) \\
f, C\end{array}$ & $\begin{array}{c}3.57(0.11) \\
C, E\end{array}$ & $\begin{array}{c}2.48(0.00) \\
b, A\end{array}$ & $\begin{array}{c}5.39(0.09) \\
e, G\end{array}$ \\
\hline 8 & $\begin{array}{c}3.29(0.11) \\
f, B\end{array}$ & $\begin{array}{c}3.85(0.02) \\
f, D\end{array}$ & $\begin{array}{c}3.92(0.03) \\
d, D\end{array}$ & $\begin{array}{c}3.30(0.05) \\
f, B\end{array}$ & $\begin{array}{c}3.83(0.03) \\
\mathrm{d}, \mathrm{D}\end{array}$ & $\begin{array}{c}3.70(0.04) \\
g, C\end{array}$ & $\begin{array}{c}3.90(0.09) \\
\text { d,D }\end{array}$ & $\begin{array}{c}3.00(0.01) \\
\mathrm{C}, \mathrm{A}\end{array}$ & $\begin{array}{c}6.84(0.06) \\
f, E\end{array}$ \\
\hline
\end{tabular}

Each value is the mean of three replicates; Standard deviation values are given in parentheses; Means presented in the same column followed by different lowercase letters are statistically different $(\mathrm{P}<0.05)$; Means in the same row followed by different capital letters are statistically different $(P<0.05)$; NB - nutrient broth

Similar tendency was observed in the experiment with $L$. monocytogenes, where lag phase practically lasted $8 \mathrm{~h}$. DM exibited stronger antibacterial effect toward $L$. 
monocytogenes under the experimental conditions used. It was especially clearly visible after the first hour of incubation, when its count was significantly reduced in all samples.

Tidona et al. (2011) reported inhibitory activity of undigested and digested DM against $L$. monocytogenes in a dose dependent manner, while Nazzaro et al. (2010) showed antibacterial activity of hydrolyzed DM toward $S$. aureus. The antimicrobial activity of DM is explained by its high concentration of lysozyme (Coppola et al., 2002; Vincenzetti et al., 2008; Zhang et al., 2008). This protein exibits strong antibacterial activity, particularly toward Gram positive bacteria. LYZ belongs to a family of enzymes which cleave the b1,4 linkages between $\mathrm{N}$-acetylmuramic acid and 2-acetylamino-2-deoxy-D-glucose residues in mucopolysaccharide or mucopeptide components in cell walls (Floris et al., 2003). Gram negative bacteria are less susceptible to its activity owing to their outer layer which does not allow the entry of LYZ moleculs to the target places in peptidoglycan structure (Floris et al., 2003). The obtained different values of $L Y Z$ content in tested milk samples (Table 3) can be due to different lactation periods (65-210 days pp.) of the donkeys and to different analytical methods applied (Salimei \& Fantuz, 2012).

Therefore, literature data about the $L Y Z$ content in DM ranges from $1 \mathrm{~g} / \mathrm{L}$ (Vincenzetti et al., 2008) to $4 \mathrm{~g} / \mathrm{L}$ (Coppola et al., 2002). The relationship between LYZ content and antibacterial activity of DM samples toward tested bacteria was not established in this investigation, since DM samples with $L Y Z$ content ranging from 0.67 to $3.54 \mathrm{~g} / \mathrm{L}$ showed similar effect against $L$. monocytogenes, while sample $\mathrm{H}(1.24 \mathrm{~g} / \mathrm{L}$ of $\mathrm{LYZ})$ had stronger activity against $S$. aureus compared to other samples with higher LYZ content. This probably indicates that $L Y Z$ is not the only antimicrobial agent in DM which can act against tested Gram positive bacteria, since it is a complex medium with numerous different compounds which could contribute to the overal antimicrobial potential. Among those compounds are defi- nitely certain fatty acids, which composition in the tested DM samples is presented in Table 3.

Main determinated fatty acids with well known antibacterial activity toward Gram positive bacteria (Galbraith et al., 1971; Galbraith \& Miller, 1973) were linoleic acid $\left(\mathrm{C}_{18: 2}\right)$, lauric acid $\left(\mathrm{C}_{12: 0}\right)$ and oleic acid $\left(\mathrm{C}_{18: 1}\right)$ (Table 3 ), which account from 40.3 to $54.7 \%$ of the total fatty acids content in analysed milk samples. These long chain fatty acids $\left(\mathrm{C}_{18: 2}, \mathrm{C}_{18: 1}\right)$ stimulated oxygen uptake by Gram positive bacteria at bactericidal and protoplast lytic concentrations and produced inhibition at higher levels (Galbraith \& Miller, 1973). Linolenic acid caused an increase in lag phase duration of Gram positive bacteria (Galbraith et al., 1971). The palmitic acid $\left(\mathrm{C}_{16: 0}\right)$ was also detected in high contentration in all tested milk samples, but there is no data on its antimicrobial action against $L$. monocytogenes or $S$. aureus.

Although fatty acids probably play a supporting role in the total antimicrobial activity of DM it would be useful to investigate their individual contribution to this activity, especially if it is known that calcium and magnesium ions, also present in DM, reverse their activities.

Since this experiment was performed at 38 ${ }^{\circ} \mathrm{C}$ which is the donkeys' body temperature (Etana et al., 2011), antimicrobial agents will not allow quick growth of L. monocytogenes and $S$. aureus in milk, if it is not cooled imidiately.

However, DM at applied level of contamination did not possess any bactericidal activity against tested bacteria. Therefore, their presence and number in raw DM will depend on the health of the mammary gland and hygienic milking procedures applied.

DM could be effective in the treatment of listeriosis and staphylococcosis, since Tidona et al. (2011) reported high resistance of DM LYZ to the degradation by gastrointestinal enzymes, while Nazzaro et al. (2010) detected the presence of compounds in hydrolyzed DM, different from $L Y Z$, capable of inhibiting the growth of $S$. aureus. 
Table 3.

Fatty acid composition $(\% \mathrm{~m} / \mathrm{m})$ and lysozyme content $(\mathrm{g} / \mathrm{L})$ of the investigated donkey milk samples

\begin{tabular}{|c|c|c|c|c|c|c|c|c|}
\hline \multirow{2}{*}{$\begin{array}{l}\text { Fatty } \\
\text { acid } \\
(\% \mathrm{~m} / \mathrm{m}) \\
\end{array}$} & \multicolumn{8}{|c|}{ Donkey milk samples } \\
\hline & A & B & C & D & $E$ & $\mathbf{F}$ & $\mathbf{G}$ & $\mathbf{H}$ \\
\hline \multirow{2}{*}{ 8:0 } & $2.2(0.12)$ & $2.8(0.19)$ & $4.2(0.13)$ & $1.5(0.14)$ & $2.6(0.21)$ & $2.1(0.24)$ & $4.0(0.10)$ & $3.7(0.34)$ \\
\hline & b & $\mathrm{C}$ & e & a & $\mathrm{C}$ & $\mathrm{b}$ & e & $d$ \\
\hline \multirow{2}{*}{$10: 0$} & $5.3(0.37)$ & $12.8(1.28)$ & $11.3(0.59)$ & $4.8(0.34)$ & $9.3(0.33)$ & $5.4(0.25)$ & $11.0(0.18)$ & $13.8(0.99)$ \\
\hline & $\begin{array}{c}a \\
0.9(0.04)\end{array}$ & $\begin{array}{c}d \\
2.1(0.19)\end{array}$ & $\begin{array}{c}\mathrm{C} \\
1.1(0.15)\end{array}$ & $\begin{array}{c}a \\
0.8(0.05)\end{array}$ & $\begin{array}{c}b \\
1.9(0.12)\end{array}$ & $\begin{array}{c}\mathrm{a} \\
1.1(0.12)\end{array}$ & $\begin{array}{c}C \\
2.1(0.16)\end{array}$ & $\begin{array}{c}d \\
2.2(0.17)\end{array}$ \\
\hline $11: 0$ & a & b & a & - & b & a & b & $\mathrm{b}$ \\
\hline $12: 0$ & $\begin{array}{c}6.1(0.42) \\
b\end{array}$ & $\begin{array}{c}15.7(0.98) \\
\mathrm{e}\end{array}$ & $\begin{array}{c}10.6(0.20) \\
C\end{array}$ & $\begin{array}{c}4.8(0.15) \\
a\end{array}$ & $\begin{array}{c}13.3(1.19) \\
d\end{array}$ & $\begin{array}{c}6.2(0.39) \\
b\end{array}$ & $\begin{array}{c}11.8(0.72) \\
C\end{array}$ & $\begin{array}{c}14.2(0.12) \\
d\end{array}$ \\
\hline $14: 0$ & $\begin{array}{c}6.3(0.42) \\
b\end{array}$ & $\underset{f}{13.5(0.94)}$ & $\begin{array}{c}8.6(0.31) \\
c\end{array}$ & $\begin{array}{c}5.1(0.41) \\
a\end{array}$ & $\underset{f}{13.3(0.81)}$ & $\begin{array}{c}6.6(0.36) \\
b\end{array}$ & $\begin{array}{c}10.7(0.86) \\
\mathrm{e}\end{array}$ & $\begin{array}{c}12.2(0.86) \\
\mathrm{e}\end{array}$ \\
\hline $14: 1$ & $\begin{array}{c}0.2(0.03) \\
b\end{array}$ & $\underset{f}{0.9}(0.04)$ & $\begin{array}{c}\text { n.d. } \\
\text { a }\end{array}$ & $\begin{array}{c}0.5(0.01) \\
\mathrm{e}\end{array}$ & $\begin{array}{c}1.1(0.05) \\
g\end{array}$ & $\begin{array}{c}0.4(0.03) \\
d\end{array}$ & $\begin{array}{c}0.2(0.03) \\
c\end{array}$ & $\begin{array}{c}\text { n.d. } \\
\text { a }\end{array}$ \\
\hline $15: 0$ & $\begin{array}{c}1.0(0.08) \\
\mathrm{e}\end{array}$ & $\begin{array}{c}0.5(0.03) \\
b\end{array}$ & $\begin{array}{c}0.8(0.09) \\
d\end{array}$ & $\begin{array}{c}0.6(0.03) \\
C\end{array}$ & $\begin{array}{c}0.8(0.06) \\
\mathrm{de}\end{array}$ & $\begin{array}{c}0.8(0.09) \\
d\end{array}$ & $\begin{array}{l}\text { n.d. } \\
\text { a }\end{array}$ & $\begin{array}{c}\text { n.d. } \\
\text { a }\end{array}$ \\
\hline $16: 0$ & $\begin{array}{c}19.4(0.95) \\
d\end{array}$ & $\begin{array}{c}17.8(0.09) \\
b\end{array}$ & $\begin{array}{c}16.0(0.58) \\
a\end{array}$ & $\begin{array}{c}18.1(0.14) \\
\text { bc }\end{array}$ & $\begin{array}{c}18.5(0.64) \\
\text { bcd }\end{array}$ & $\begin{array}{c}19.2(0.46) \\
\mathrm{cd}\end{array}$ & $\begin{array}{c}18.3(1.14) \\
\text { bcd }\end{array}$ & $\begin{array}{c}18.8(0.50) \\
\mathrm{cd}\end{array}$ \\
\hline $16: 1$ & $\begin{array}{c}6.6(0.46) \\
d\end{array}$ & $\begin{array}{c}4.1(0.31) a \\
b\end{array}$ & $\begin{array}{c}6.3(0.59) \\
d\end{array}$ & $\begin{array}{c}6.1(0.14) \\
d\end{array}$ & $\begin{array}{c}6.6(0.66) \\
d\end{array}$ & $\begin{array}{c}5.2(0.15) \\
\mathrm{C}\end{array}$ & $\begin{array}{c}3.5(0.04) \\
a\end{array}$ & $\begin{array}{c}4.3(0.42) \\
b\end{array}$ \\
\hline 18:0 & $\begin{array}{c}1.3(0.15) \\
b\end{array}$ & $\begin{array}{c}1.9(0.28) \\
d\end{array}$ & $\begin{array}{c}1.6(0.01) \\
C\end{array}$ & $\begin{array}{c}1.4(0.13) b \\
c\end{array}$ & $\begin{array}{c}1.5(0.05) \\
b c\end{array}$ & $\begin{array}{c}1.5(0.06) \\
b c\end{array}$ & $\begin{array}{c}1.0(0.10) \\
a\end{array}$ & $\begin{array}{c}1.6(0.21) \\
C\end{array}$ \\
\hline $18: 1$ & $\begin{array}{c}20.1(0.90) \\
d\end{array}$ & $\begin{array}{c}10.9(1.13) \\
a\end{array}$ & $\begin{array}{c}13.1(0.16) \\
b\end{array}$ & $\begin{array}{c}20.9(0.08) \\
d\end{array}$ & $\begin{array}{c}11.8(1.04) \\
a b\end{array}$ & $\begin{array}{c}20.3(1.35) \\
d\end{array}$ & $\begin{array}{c}15.0(0.55) \\
C\end{array}$ & $\begin{array}{c}12.3(1.22) \\
a b\end{array}$ \\
\hline $18: 2 n 6$ & $\begin{array}{c}24.3(0.52) \\
\mathrm{e}\end{array}$ & $\begin{array}{c}14.4(1.37) \\
b\end{array}$ & $\begin{array}{c}18.9(0.47) \\
C\end{array}$ & $\underset{f}{28.7(0.49)}$ & $\begin{array}{c}15.8(0.42) \\
b\end{array}$ & $\begin{array}{c}21.9(0.90) \\
d\end{array}$ & $\begin{array}{c}18.1(0.30) \\
C\end{array}$ & $\begin{array}{c}12.6(1.54) \\
a\end{array}$ \\
\hline $18: 3 n 3$ & $\begin{array}{c}6.3(0.11) \\
d\end{array}$ & $\begin{array}{c}2.6(0.07) \\
a\end{array}$ & $\begin{array}{c}7.6(0.62) \\
\mathrm{e}\end{array}$ & $\begin{array}{c}6.5(0.15) \\
d\end{array}$ & $\begin{array}{c}3.4(0.38) \\
b\end{array}$ & $\begin{array}{c}8.6(0.48) \\
f\end{array}$ & $\begin{array}{c}4.1(0.34) \\
C\end{array}$ & $\begin{array}{c}4.4(0.21) \\
C\end{array}$ \\
\hline 20:2 & $\begin{array}{c}\text { n.d. } \\
\text { a }\end{array}$ & $\begin{array}{c}\text { n.d. } \\
\text { a }\end{array}$ & $\begin{array}{c}\text { n.d. } \\
\text { a }\end{array}$ & $\begin{array}{c}\text { n.d. } \\
\text { a }\end{array}$ & $\begin{array}{c}\text { n.d. } \\
\text { a }\end{array}$ & $\begin{array}{c}0.8(0.08) \\
b\end{array}$ & $\begin{array}{c}\text { n.d. } \\
\text { a }\end{array}$ & $\begin{array}{c}\text { n.d. } \\
\text { a }\end{array}$ \\
\hline $\begin{array}{l}\text { LYZ } \\
\text { content } \\
(\mathrm{g} / \mathrm{L})\end{array}$ & $\begin{array}{c}3.52 \\
(0.08) \\
e\end{array}$ & $\begin{array}{c}3.54 \\
(0.09) \\
e\end{array}$ & $\begin{array}{c}3.42 \\
(0.06) \\
e\end{array}$ & $\begin{array}{c}1.3 \\
(0.05) \\
b\end{array}$ & $\begin{array}{c}0.67 \\
(0.05) \\
a\end{array}$ & $\begin{array}{c}2.52 \\
(0.07) \\
d\end{array}$ & $\begin{array}{c}1.74 \\
(0.06) \\
C\end{array}$ & $\begin{array}{c}1.24 \\
(0.07) \\
b\end{array}$ \\
\hline
\end{tabular}

Each value is the mean of three replicates. Standard deviation values are given in parentheses. Means presented in the same line followed by different lower case are statistically different $(\mathrm{P}<0.05)$

\section{CONCLUSIONS}

The detected antibacterial action of DM against $L$. monocytogenes and $S$. aureus increased in bacterial lag phase duration as well as inhibited their growth. Under these experimental conditions, DM showed stronger antibacterial potential against $L$. monocytogenes. LYZ could be marked as the major antibacterial agent in $D M$, since it was present in high concentration. Different values of LYZ content determinated in this study could be explained by the different lactation stages milking animals belong to. However, the absence of established links between antibacterial activity of DM and its LYZ content could indicate existance of some other compounds in DM which additionaly contribute to the total antibacterial activity of
DM against tested bacteria. Among the detected fatty acids with known antibacterial activity, the most abundant in DM were linoleic, lauric and oleic acid.

\section{ACKNOWLEDGMENTS}

This work is a part of the National Project (TR-31029) financially supported by the Ministry of Education, Science and Technological Development, Republic of Serbia. Authors are grateful to Slobodan Simić and Nikola Nilić (Special Nature Reserve "Zasavica", Serbia) for providing the milk samples.

\section{REFERENCES}

1. Blench, R. (1999). Traditional livestock breeds: geographical distribution and dynamics in relation to the ecology of West Africa. (http://www.odi.org.uk/publications/2041traditional-livestock-breeds-geographical- 
distribution-dynamics-relations-ecology-westafrica)

2. Brumini, D., Furlund, C. B., Comi, I., Devold, T. G., Marletta, D., Vegarud, G. E., Jonassen, C. M. (2013). Antiviral activity of donkey milk protein fractions on echovirus type 5. International Dairy Journal, 28, 109-111.

3. Conte, F., Scatassa, M. L., Monsu, G., Lo Verde, V., Finocchiaro, A., De Fino, M. (2006). Monitoring of safety and quality of donkey`s milk. In F. J. M. Smulders (Ed.), Food safety assurance and veterinary public health: Towards a risk-based chain control ( $1^{\text {st }}$ ed.) Wageningen, The Netherlands: Wageningen Academic Publishers.

4. Coppola, R., Salimei, E., Succi, M., Sorrentino, E., Nanni, M., Ranieri, P. (2002). Behaviour of Lactobacillus rhamnosus strains in ass's milk. Annals of Microbiology, 52, 55-60.

5. ECSCEE. (2013). Easthorn Clinical Services in Central and Eastern Europe, Serbia (http://www.easthorn.com/geographicalreach/serbia)

6. EN ISO. (2004). Microbiology of food and animal feeding stuffs - Horizontal method for the detection and enumeration of Listeria monocytogenes - Part 1: Detection method. ISO standard 11290-1:1996/A1:2004. Geneva, Switzerland: International Organization for Standardization.

7. Etana, M., Endeabu, B., Jenberie, S., \& Negussie, H. (2011). Determination of reference physiological values for working donkeys of Ethiopia. Ethiopian Veterinary Journal, 15, 7986.

8. FAO DAD-IS. (2009). Domestic animal diversity information system: breeds. (http://dad.fao.org)

9. FASFC (2013). Advice 11-2013 of the Scientific Committee of the FASFC on the evaluation of the risks and benefits of the consumption of raw milk from animal species other than cows (http://www.favv.be/scientificcommittee/advices/ documents/Advice11-2013.pdf)

10. Floris, R., Recio, I., Berkhout, B., Visser, S. (2003). Antibacterial and antiviral effects of milk proteins and derivatives thereof. Current Pharmaceutical Design, 9, 1257-1275.

11. Galbraith, H., Miller, T. B., Paton, A. M., Thompson, J. K. (1971). Antibacterial Activity of Long Chain Fatty Acids and the Reversal with Calcium, Magnesium, Ergocalciferol and Cholesterol. Journal of Applied Microbiology, 34, 803-813.

12. Galbraith, H. \& Miller, T. B. (1973). Effect of long chain fatty acids on bacterial respiration and amino acid uptake. Journal of Applied Bacteriology, 36, 659.

13. Havemose, M., Weisbjerg, M., Bredie, W., Neilsen, J. (2004): Influence of feeding different types of roughage on the oxidative stability of milk. International Dairy Journal, 14, 563-570.

14. Iacono, G., Carroccio, A., Cavataio, F., Montalto, G., Soresi, M., Balsamo, V. (1992). Use of ass's milk in multiple food allergy. Journal of
Pediatric Gastroenterology and Nutrition, 14, 177-181.

15. ISO. (2003). Microbiology of food and animal feeding stuffs - Horizontal method for the enumeration of coagulase-positive staphylococci (Staphylococcus aureus and other species) Part 1: Technique using BairdeParker agar medium. ISO standard 6888-1:1999/A1:2003. Geneva, Switzerland: International Organization for Standardization.

16. Kravić, S., Marjanović, N., Suturović, Z., ŠvarcGajić, J., Stojanović, Z., Pucarević, M. (2010). Determination of trans fatty acid content of Serbian shortening by gas chromatography-mass spectrometry. Acta Alimentaria, 39, 413-423.

17. Kugler, W., Grunenfelder, H. P., \& Broxham, E. (2008). Donkey breeds in Europe: inventory, description, need for action, conservation: report 2007/2008. URL.

http://www.save-foundation.net/pdf/donkey.pdf

18. Mao, X., Gu, J., Sun, Y., Xu, S., Zhang, X., Yang, H., Ren, F. (2009). Anti-proliferative and anti-tumour effect of active components in donkey milk on A549 human lung cancer cells. International Dairy Journal, 19, 703-708

19. Monti, G., Bertino, E., Muratore, M. C., Coscia, A., Cresi, F., Silvestro, L., et al. (2007). Efficiacy of donkey's milk in treating highly problematic cow's milk allergic children: an in vivo and in vitro study. Pediatric Allergy and Immunology, 18, 258-264.

20. Nazzaro, F., Orlando, P., Fratianni, F., Coppola, R. (2010). Isolation of Components with Antimicrobial Property from the Donkey Milk: A Preliminary Study. The Open Food Science Journal, 4, 43-47.

21. Salimei, E., Fantuz, F., Coppola, R., Chiofalo, B., Polidori, P., Varisco, G. (2004). Composition and characteristics of ass's milk. Animal Research, 53, 67-78.

22. Salimei, E., \& Fantuz, F. (2012). Equid milk for human consumption. International Dairy Journal, 24, 130-142.

23. Šarić, Ć. LJ., Šarić, M. B., Mandić, I. A., Torbica, M. A., Tomić, M. J., Cvetković, D. D., Okanović, G. Đ. (2012). Antibacterial properties of Domestic Balkan donkeys' milk. International Dairy Journal, 25, 142-146.

24. Sarno, E., Santoro, A. M. L., Di Palo, R., Costanzo, N. (2012). Microbiological quality of raw donkey milk from Campania Region. Italian Journal of Animal Science, 11, 266-269.

25. Tafaro, A., Magrone, T., Jirillo, F., Martemucci, G., D'Alessandro, A. G., Amati, L., Jirillo, E. (2007). Immunological properties of donkey's milk: its potential use in the prevention of atherosclerosis. Current Pharmaceutical Design, 13, 3711-7.

26. Tidona, F., Sekse, C., Criscione, A., Jacobsen, M., Bordonaro, S., Marletta, D., et al. (2011). Antimicrobial effect of donkeys' milk digested in 
vitro with human gastrointestinal enzymes. International Dairy Journal, 21, 158-165.

27. Vincenzetti, S., Polidori, P., Mariani, P., Cammertoni, N., Fantuz, F., \& Vita, A. (2008). Donkey's milk protein fractions characterization. Food Chemistry, 106, 640-649.

28. Vita, D., Passalacqua, G., Di Pasquale, G., Caminiti, L., Crisafulli, G., Rulli, I., et al. (2007).
Ass's milk in children with atopic dermatitis and cow's milk allergy: crossover comparison with goat's milk. Pediatric Allergy and Immunology, 18, 594-598.

29. Zhang, X. Y., Zhao, L., Jiang, L., Dong, M. L., Ren, F. Z. (2008). The antimicrobial activity of donkey milk and its microflora changes during storage. Food Control, 19, 1191-1195.

\section{АНТИБАКТЕРИЈСКА АКТИВНОСТ МЛЕКА МАГАРИЦЕ РАСЕ ДОМАЋИ БАЛКАНСКИ МАГАРАЦ НА Listeria monocytogenes И Staphylococcus aureus}

Љубиша Ћ. Шарић ${ }^{1,}$, Бојана М. Шарић ${ }^{1}$, Снежана Ж. Кравић ${ }^{2}$, Драгана В. Плавшић ${ }^{1}$, Иван Љ. Миловановић ${ }^{1}$, Јасмина М. Губић ${ }^{1}$, Наташа М. Недељковић ${ }^{1}$

${ }^{1}$ Универзитет у Новом Саду, Научни институт за прехрамбене технологије у Новом Саду, Булевар цара Лазара 1, 21000 Нови Сад, Србија

${ }^{2}$ Универзитет у Новом Саду, Технолошки фракултет, Булевар цара Лазара 1 , 21000 Нови Сад, Србија

Сажетак: Циљ овог истраживања био је испитивање антибактеријске активности сировог млека магарице расе Домаћи балкански магарац, на L. monocytogenes и S. aureus. Испитивање антибактеријске активности је извршено на вештачки контаминираним узорцима млека, праћењем промена броја испитиваних бактерија током 8 сати инкубирања на температури од $38{ }^{\circ} \mathrm{C}$. Такође је одређен садржај лизозима и масних киселина у млеку магарице. Испитивање вештачки контаминираних узорака је показало инхибиторни ефекат млека магарице на обе бактеријске врсте. Садржај лизозима у анализираним узорцима млека је био у интервалу од 0.67 до $3.54 \mathrm{~g} / \mathrm{L}$. Од масних киселина са познатом антибактеријском активношћу на Грам позитивне бактерије, у највећој концентрацији су биле присутне линолна, олеинска и лауринска киселина.

Кључне речи: млеко магарице, антибактеријска активност, L. monocytogenes, S. aureus

Received: 19 February 2014

Accepted: 22 April 2014 